\title{
GIOVANI, ELENA; GAMBIER, YVES (ED.). RECEPTION STUDIES AND AUDIOVISUAL TRANSLATION. AMSTERDAM/PHILADELPHIA: JOHN BENJAMINS PUBLISHING COMPANY, 2018. 353P.
}

\section{Marileide Dias Esqueda* Igor Antonio Lourenço da Silva**}

Foi com grande interesse, e por duas razões principais, que realizamos a leitura da obra Reception Studies and Audiovisual Translation, publicada em 2018 e organizada por Elena Di Giovanni, Professora Associada da Universidade de Macerata, na Itália, e Yves Gambier, Professor Emérito da Universidade de Turku, na Finlândia.

Já que toda tradução prospecta determinado tipo de público, nosso interesse, na qualidade de pesquisadores dos Estudos da Tradução, recai, inevitavelmente, nos estudos que lidam com a necessidade e, ao mesmo tempo, na dificuldade de construir instrumentos de pesquisa que possam aferir os hábitos, as preferências, as expectativas e as necessidades do público-alvo com relação aos materiais traduzidos. De fato, na obra organizada por Di Giovanni e Gambier, é possível encontrar descrições de vários instrumentos de pesquisa dedicados à aferição dos elementos que influenciam a recepção de um produto traduzido, como idade, sexo, nível de escolaridade, identidade linguística e comportamento dos espectadores. Esses elementos, de acordo com os estudos de vários dos autores que compõem a obra, têm forte impacto na maneira como os textos são traduzidos e posteriormente recebidos.

Uma vez engajados na formação de tradutores, nosso interesse também incide em como os estudos da recepção de textos traduzidos podem orientar melhor o ensino e a aprendizagem de tradução. A partir das contribuições contidas na obra de Di Giovani e Gambier, sobretudo com relação às formas como os materiais traduzidos para as diversas mídias digitais, para o cinema e para a televisão vêm sendo recebidos pelos usuários, podemos vislumbrar o planejamento de aulas, disciplinas ou cursos mais próximos das realidades tanto mercadológicas quanto acadêmicas e, assim, formar profissionais mais bem preparados, que valorizem as necessidades e preferências do público-alvo e que avaliem como as novas relações

\footnotetext{
* Universidade Federal de Uberlândia (UFU), Uberlândia, MG, Brasil. marileide.esqueda@ufu.br, https://orcid.org/0000-0002-6941-7926

** Universidade Federal de Uberlândia (UFU), Uberlândia, MG, Brasil. ials@ufu.br, https://orcid. org/0000-0003-0738-3262
} 
midiáticas propiciadas pela atual miríade tecnológica e pela internet vêm afetando o trabalho dos tradutores.

Assim, explorando os estudos de recepção na subárea da tradução audiovisual, a obra de Di Giovanni e Gambier atende aos nossos interesses e, certamente, aos de outros pesquisadores e professores. Os diversos produtos audiovisuais, as tecnologias e os instrumentos de aferição das preferências dos espectadores tratados na obra mostram que a tradução audiovisual é uma subárea que impulsiona as tendências de pesquisa sobre a recepção de traduções. Após a leitura dessa obra, pesquisadores, professores, estudantes e profissionais dos Estudos da Tradução poderão estar menos propensos às especulações sobre as reações do público aos materiais traduzidos.

Na primeira parte da obra, intitulada Defining reception studies, encontram-se três capítulos. No primeiro, Media audiences and reception studies, Annette Hill, além de analisar os diferentes paradigmas relacionados aos estudos midiáticos, examina o drama nórdico do gênero noir Bron / Broen (The Bridge), cuja primeira cena exibe um corpo, composto de duas vítimas, estendido na fronteira entre a Suécia e a Dinamarca. O drama foi traduzido e distribuído em 157 países pela Netflix. Interessados em analisar como os espectadores de duas culturas diferentes se envolvem no drama, a autora e sua equipe realizam uma pesquisa qualitativa, de abordagem pragmática, que faz uso de várias técnicas de pesquisa, como entrevistas com diretores e observações da reação de alguns espectadores. A autora conclui que o drama é uma maneira de construir diferenças culturais e linguísticas e revela semelhanças nos imaginários tanto de dinamarqueses quanto de suecos. Para Hill, os espectadores se envolvem no drama do crime e percebem criticamente suas realidades sociais.

Concebido como uma revisão da literatura e intitulado Film, cinema and reception studies: revisiting research on audience's filmic and cinematic experiences, o capítulo de Daniel Biltereyst e Philippe Meers distingue o filme do cinema. De acordo com os autores, define-se o primeiro como um produto social que desencadeia a imaginação do público e o segundo como uma instituição social e cultural, ou seja, uma rede ampliada de atividades em torno dos filmes (i.e., produção, distribuição, exibição, consumo). Os autores integram as duas noções a fim de definir novos caminhos para os estudos de recepção, cujo ponto de partida principal é considerar o papel ativo do espectador como aquele que constrói os significados.

Translation studies, audiovisual translation and reception é o título do capítulo escrito por Yves Gambier. O autor, depois de mencionar as contribuições de Nida (1964), Gutt (1991/2000), Even-Zohar (1990), Lefevere (1992/2017), Toury 
(1995/2012), Chesterman (1993) e Scott (2012), enfatiza que a produção de uma tradução sempre esteve ligada às reações do público-alvo e confirma a necessidade de aprofundamento dos estudos de recepção. Esclarecendo as ambiguidades relacionadas às noções de percepção e recepção, sendo a primeira uma série de opiniões e impressões e a segunda uma maneira de estudar as formas como os produtos audiovisuais são apreciados pelo público, o autor se baseia em Kovačič (1995), Chesterman $(1998$; 2007) e Gambier (2009) para adotar o modelo triplo "resposta, reação e repercussão". Esse modelo também é discutido no capítulo escrito por Tiina Tuominen, conforme esclarecido a seguir.

$\mathrm{Na}$ segunda parte do livro, Methodology in reception studies and audiovisual translation, encontram-se quatro capítulos. No primeiro, Multi-method research: reception in context, Tiina Tuominen discute as abordagens de pesquisa que se dedicam à recepção de produtos audiovisuais. A autora afirma que a tradução por si só não produz a experiência da recepção, a qual é construída em colaboração com o público e mediante interações anteriormente estabelecidas. Com base nas categorias de Chesterman, que incluem "resposta" (i.e., capacidade cognitiva do espectador em seguir a tradução), "reação" (i.e., interpretação do leitor do que ele lê na tradução) e "repercussão" (i.e., avaliação do produto traduzido pelo espectador e pela sociedade como um todo), Tuominen explica que a ampla variedade de estudos e abordagens dos estudos de recepção permite o compartilhamento de métodos de reação e repercussão relacionados à vida e aos contextos sociais aos quais pertencem o público.

O capítulo de Jan-Luis Kruger e Stephen Doherty, Triangulation of online and offline measures of processing and reception in $A V T$, centra-se nas aferições psicológicas, psicométricas e de desempenho, tanto utilizadas de forma isolada quanto em combinação, para fornecer uma síntese do que vem sendo realizado nos estudos de recepção de produtos audiovisuais. Os autores relatam as formas de aferição e processamento off-line, que compreendem o Rappel, o NASA-Task Load index e a escala Likert, para aferir o nível de imersão psicológica do espectador a partir de diferentes métodos de tradução audiovisual. Também discutem sobre os instrumentos online, que geralmente incluem rastreamento ocular, eletroencefalografia, reação eletrodérmica / reflexo psicogalvânico e frequência cardíaca. Dessa forma, o capítulo oferece uma visão geral das diferentes formas e instrumentos para estudar a recepção na tradução audiovisual.

Roberto A. Valdeón explora as abordagens discursiva, pragmática e multimodal dos estudos de recepção relacionados à tradução audiovisual. Seu capítulo, Discourse analysis, pragmatics, multimodal analysis, visa chamar a atenção para 
o fato de que a tradução de produtos audiovisuais deve estar preocupada não só com os estudos de recepção, mas também, numa perspectiva mais ampla, com a inter-relação entre os Estudos da Imagem (Imagology ou Image Studies) e os Estudos da Tradução. Com base no trabalho de Kuran-Burçoglu (2000), Van Doorslaer, Flynn e Leerssen (2016), Kress e Van Leeuwen (2007) sobre como ler uma imagem, Valdeón salienta a necessidade de pesquisas sobre as complexidades cultural e linguística da comunicação visual.

No capítulo Historical approaches to AVT reception: methods, issues and perspectives, Serenella Zanotti parte dos estudos históricos de recepção de filmes para enfatizar que os diversos meios utilizados por Hollywood para facilitar a recepção de suas produções nos mercados estrangeiros passam pela tradução, ou seja, a principal preocupação da indústria cinematográfica de Hollywood é a resposta do público. Para a autora, a pesquisa histórica sobre a tradução audiovisual ainda é incipiente e o que tem sido escrito atualmente sobre a recepção do público é frequentemente filtrado pela indústria cinematográfica estadunidense.

$\mathrm{Na}$ terceira parte do livro, AVT modalities and reception studies, encontram-se quatro capítulos. No primeiro, Dubbing, perception and reception, Elena Di Giovanni observa que, embora seja uma das técnicas mais antigas utilizadas nas exibições de filmes, a dublagem é subestimada pelos pesquisadores. A autora fornece uma visão geral de abordagens, ferramentas e métodos que podem ser usados para a pesquisa sobre dublagem com foco na recepção, particularmente em relação a filmes e produtos dirigidos à televisão, a fim de estabelecer o nível de compreensão e participação do público. Para Di Giovanni, nos cinco continentes, a dublagem é a única forma de tradução audiovisual destinada aos jovens espectadores quando se trata de entretenimento infantil. Os estudos sobre a dublagem são essencialmente comparativos, com foco em soluções linguísticas, transferência de referências culturais e questões de sincronia labial; no entanto, Di Giovanni destaca seu potencial para a pesquisa interdisciplinar. A autora ainda examina as contribuições dos últimos 15 anos de estudos dedicados a recepção e percepção de textos audiovisuais dublados.

Para Kristijan Nikolić, encontrar os temas certos, usar a tecnologia adequada e obter financiamentos para a condução de pesquisas são os problemas que historicamente dificultam os estudos de recepção na tradução audiovisual. Seu capítulo, Reception studies in audiovisual translation - interlingual subtitling, examina as origens e os desenvolvimentos futuros desses estudos. Devido à vulnerabilidade da legendagem, essencialmente construída com base em formas condensadas das palavras faladas no material-fonte, Nikolić ressalta que a redução pode levar 
o espectador a acreditar que o tradutor tenha omitido algumas informações nas legendas, o que justifica o estudo da legendagem interlinguística em estudos de recepção. Nesse capítulo, além de discutir como os métodos de pesquisa que utilizam as técnicas de rastreamento ocular combinadas com a análise estatística influenciam os estudos de recepção, Nikolić mostra a importância da pesquisa sobre o uso de legendagem interlinguística e suas consequências para a aprendizagem de línguas.

No capítulo Reception studies in live and pre-recorded subtitles for the deaf and hard of bearing, Pablo Romero-Fresco, partindo de uma abordagem histórica, refere-se à primeira tese sobre como criar legendas para surdos publicada nos anos de 1940 e à implementação desse tipo de legendagem nos anos de $1950 \mathrm{em}$ programas televisivos exibidos em uma associação para surdos em Boston, nos Estados Unidos. De acordo com Romero-Fresco, as formas de exibição de legendas variam consideravelmente de país para país e são acompanhadas por pesquisas sobre a recepção que se revelam essenciais para informar e moldar as práticas dos dias de hoje. Para o autor, foi na década de 1990, com o trabalho pioneiro de Linde e Kay (1990), que o uso da tecnologia de rastreamento ocular passou a servir como ferramenta para se estudar a velocidade da exibição das legendas e seu processo de leitura. Nessa direção, vale ressaltar que, embora negligenciado pelo autor, o trabalho pioneiro de Franco e Araújo (2003) no Brasil revela dados empíricos igualmente importantes para melhor adequar as legendas para surdos e ensurdecidos dos programas de uma das emissoras de televisão mais influentes do país, a Rede Globo.

Em seu segundo capítulo na obra, com o título Audio description and reception-centered research, Elena Di Giovanni descreve a história da audiodescrição e sua recepção. Para a autora, a audiodescrição é uma ferramenta extremamente valiosa para restaurar o conhecimento e a apreciação de filmes e textos audiovisuais por cegos e deficientes visuais: traduzir signos icônicos em sequências verbais e combiná-los com a trilha sonora do filme é fornecer aos deficientes visuais os elementos necessários para sua própria interpretação e recepção. Em relação à pesquisa em audiodescrição, a autora explica que, nas últimas décadas, os esforços se multiplicaram para ampliar a oferta e melhorar a qualidade da audiodescrição no mundo. Fornecendo uma visão geral das ferramentas e métodos que os pesquisadores vêm utilizando atualmente, aponta que os estudos sobre a audiodescrição não se concentram apenas nas preferências do espectador, mas também em aferir a compreensão em diferentes níveis, como os do léxico, sintaxe e velocidade de transmissão. Segundo Di Giovanni, pelo menos quatro grandes eixos podem ser identificados nos trabalhos sobre a recepção da audiodescrição até a presente data, a saber: estudos descritivos (ênfases linguísticas 
e semânticas), pesquisas de cunho psicológico (pesquisas empíricas na inter-relação tradução audiovisual e psicologia), eixos alternativos (uso e integração de outras tecnologias para se estudar a percepção do espectador, como vozes artificiais e tecnologias texto-fala) e, finalmente, eixos inclusivos (estudos envolvendo as artes e formas entretenimento ao vivo). No que tange ao cenário brasileiro de pesquisas sobre a audiodescrição, também negligenciado nesse capítulo, destacam-se a obra pioneira de Araújo et al. (2016) e diversos estudos conduzidos pelo grupo de pesquisa LEAD (Legendagem e Audiodescrição), da Universidade Estadual do Ceará (UECE), como o de Seoane e Araújo (2016). Trata-se de uma tradição em pesquisa bastante consolidada no Brasil, capaz de ensejar e subsidiar diversos trabalhos em outros centros de pesquisa, como a monografia de Barbosa (2017), defendida na Universidade Federal de Uberlândia, com uma proposta de audiodescrição de um episódio da série BoJack Horseman. A referida proposta, orientada pelo Prof. Dr. Igor A. Lourenço da Silva, contou com bastante auxílio de Alexandra Frazão Seoane, membro do LEAD.

A quarta e última parte do livro, Hybrid media and new andiences, está organizada em quatro capítulos. No primeiro, Media interpreting: from user expectations to audience comprebension, Franz Pöchhacker analisa a evolução da pesquisa de interpretação para as diferentes mídias baseada em recepção, em primeiro lugar ressaltando que se trata de uma modalidade híbrida ainda não consolidada como área de pesquisa na tradução audiovisual. Pöchhacker argumenta que a interpretação feita para os programas de massa, em particular para a televisão, aparece como um fenômeno marginal em termos de tema de investigação. $\mathrm{O}$ autor conclui distinguindo as áreas de tradução e interpretação, os tipos de interpretação midiática e suas implicações para a comunicação, os cenários de interação e o público-alvo.

Carme Mangiron, no capítulo Reception studies in game localisation: taking stock, apresenta uma visão geral dos estudos de recepção conduzidos até o presente sobre a localização de jogos e centra-se em aspectos como a experiência dos jogadores, a qualidade das versões localizadas, as opiniões dos usuários dos jogos localizados e a recepção das legendas em jogos. Embora essas linhas de pesquisa sejam objeto de estudos importantes, Mangiron reconhece que são raras nos Estudos da Tradução. Para estudos futuros, Mangiron sugere implementação de investigações que analisem a reação do espectador quanto a suas preferências pela internacionalização ou localização. Algo nessa direção, embora não citado na obra, foi realizado por Coelho e Esqueda (2017), que investigaram como essas normas se evidenciam em comentários tecidos por gamers a respeito da versão totalmente localizada para o português do Brasil do jogo Uncharted 3: Drake's Deception. Os resultados obtidos 
por meio da análise dos comentários dos gamers e do gameplay capturado elucidaram a relação entre normas de expectativa e profissionais dos agentes envolvidos na localização e tradução desse material.

Consoante Alberto Fernández-Costales, em seu capítulo intitulado On the reception of mobile content: new challenges in audiovisual translation research, na era pós-PC, na qual as tecnologias favoreceram novas funcionalidades e alternativas para a visualização de conteúdos audiovisuais, novas linhas de pesquisa devem ser desenvolvidas. Para o autor, a internet tem sido a referência para novos modos de consumo de conteúdo digital e mídia audiovisual, bem como para o processo de democratização de dispositivos móveis. Nesse sentido, a combinação entre os serviços oferecidos via internet e os novos dispositivos móveis altera o comportamento do público, bem como os textos e suas traduções.

David Orrego-Carmona, no capítulo New audiences, international distribution, and translation, aponta que, antes da era da internet, as redes de televisão e comunicação tinham o poder de decidir sobre a programação e os horários de seus programas, e os telespectadores recebiam o conteúdo já preparado, com pouca ou nenhuma possibilidade de escolha. Com o advento da cultura participativa e da convergência de mídias desde os anos de 1980, começando com movimentos da cultura fandom e, mais tarde, abrangendo grupos maiores de usuários, a natureza do público mudou drasticamente. $\mathrm{O}$ autor também aponta que os espectadores estão trazendo suas percepções ou reações aos programas a que assistem no Twitter ou no Reddit, integrando-os a suas vidas e experiências diárias, indo além, e mesmo subvertendo, os propósitos da televisão. Para o autor, a tradução inseriu-se naturalmente nas várias mídias e é um elemento essencial da cultura participativa. Além disso, OrregoCarmona sugere que os pesquisadores se esforcem para estabelecer acordos com comunidades de legendadores voluntários e não profissionais para obter acesso a suas legendas e estudar seus hábitos e suas formas de produção.

Em suma, apontamos que os quinze capítulos da obra organizada por Di Giovanni e Gambier - escritos por especialistas em mídia e cinema, tradução audiovisual, interpretação, localização de jogos e multimodalidade - mostram que os estudos de recepção aplicados a produtos audiovisuais vão muito além de simples inserções de texto traduzidos para diferentes línguas. A compreensão e a apreciação do cinema e dos produtos audiovisuais como um todo são fenômenos sociais complexos relacionados a tradução e interpretação. Tal complexidade cresce excepcionalmente à medida que os meios se tornam cada vez mais diversos e imbricados, trazendo consigo novas formas de transmitir mensagens e novas formas de investigar seus usos, suas técnicas e suas recepções. 
Embora não apresente explicitamente vieses pedagógicos, essa obra também é destinada a professores e estudantes que se dedicam à tradução audiovisual. A obra nos faz refletir sobre como os novos modos de exibição de conteúdo, os dispositivos tecnológicos e os avanços nas formas de consumo estão mudando a maneira como ensinamos, aprendemos, pesquisamos, praticamos e consumimos a tradução audiovisual.

De certa forma, a obra também nos faz refletir sobre a repercussão das pesquisas desenvolvidas no Brasil: há já no país uma tradição de investigações sobre tradução audiovisual, a qual, contudo, foi sobremaneira negligenciada na obra, havendo uma única referência a um trabalho de Franco e Araújo (2003). Talvez seja um chamado para que repensemos nossa colocação no cenário internacional, buscando divulgar o vasto conhecimento produzido em território brasileiro. É nesse sentido que trazemos, em nossa abordagem de um ou outro capítulo de Di Giovani e Gambier (2018), pesquisas desenvolvidas por pesquisadores brasileiros e que muito poderiam enriquecer as argumentações da obra em tela. Nossa abordagem dessas pesquisas de forma alguma buscou ser exaustiva, até porque provavelmente conseguiríamos contemplar exemplos para todos os capítulos.

\section{REFERÊNCIAS}

ARAÚJO, Vera Lúcia. S.; ALVES, Soraya. F.; MAUCH, Carla; NAVES, Sylvia B. (Org.). Guia para Produções Audiovisuais Acessíveis. 1. ed. Brasília: Ministério da Cultura/Secretaria do Audiovisual, 2016. v. 1.

BARBOSA, Rayanne S. Uma proposta de tradução e outra de elaboração de roteiro de audiodescrição em português para a série animada Bojack Horseman. 2017. 78 f. Monografia (Graduação em Tradução) - Instituto de Letras e Linguística, Universidade Federal de Uberlândia, Uberlândia, 2017.

CHESTERMAN, Andrew. From 'Is' to 'Ought': Laws, Norms and Strategies in Translation Studies. Target, v. 5, n. 1, p. 1-20, 1993. doi: 10.1075/target.5.1.02che

CHESTERMAN, Andrew. Causes, Translations, Effects. Target, v. 10, n. 2, p. 201-230, 1998. doi: $10.1075 /$ target.10.2.02che

CHESTERMAN, Andrew. Bridge Concepts in Translation Sociology. In: WOLF, Michaela; FUKARI, Alexandra (Org.). Constructing a Sociology of Translation. Amsterdam/ Philadelphia: John Benjamins, 2007. p. 171-183. doi: 10.1075/btl.74.12che 
COELHO, Barbara R.; ESQUEDA, Marileide D. O inexplorado em Uncharted 3: normas profissionais x normas de expectativa. Tradução em Revista, Rio de Janeiro, v. 22 , p. $137-171,2017$.

DE LINDE, Zoé; KAY, Neil. The Semiotics of Subtitling. Manchester: St. Jerome, 1999.

EVEN-ZOHAR, Itamar. Polysystem Studies. Poetics Today, v. 11, n. 1, special issue, p. $1-268,1990$.

FRANCO, Eliana P. C.; ARAÚJO, Vera Lúcia S. Reading Television. The Translator, v. 9, n. 2, p. 249-267, 2003. Doi: $10.1080 / 13556509.2003 .10799156$

GAMBIER, Yves. Challenges in Research on Audiovisual Translation. Translation Research Project 2. Tarragona: Intercultural Studies Group, 2009.

GUTT, Ernst-August. Translation and Relevance: Cognition and Context. Oxford: Blackwell/ Manchester: St Jerome, 1991/2000.

KOVAČIČ, Irena. Reception of Subtitles. The Non-Existent Ideal Viewer. Translatio (Nouvelles de la FIT/FIT Newsletter), v. 14, n. 3-4, p. 376-383, 1995.

KRESS, Gunther; VAN LEEUWEN, Theo. Reading Images. The Grammar of Visual Design. London/New York: Routledge, 2007/1996.

KURAN-BURÇOĞLU, Nedret. At the Crossroads of Translation Studies and Imagology. In: CHESTERMAN, Andrew; SAN SALVADOR, Nuria Gallardo; GAMBIER, Yves (Ed.). Translation in Context. Amsterdam/Philadelphia: John Benjamins, 2000. p. 143 - 150. doi: $10.1075 /$ btl.39.16kur

LEFEVERE, André. Translation, Rewriting and the Manipulation of Literary Frame. London/New York: Routledge, 1992/2017.

NIDA, Eugene. Toward a Science of Translating. Leiden: E.J. Brill, 1964.

SCOTT, Clive. Translating the Perception of Text. Literary Translation and Phenomenology. Oxford: Legenda - Modern Humanities Research Association, 2012.

SEOANE, Alexandra Frazão; ARAÚJO, Vera Lúcia. S. A avaliação da priorização de informação em roteiros de audiodescrição: uma proposta metodológica com o rastreador ocular. In: ADERALDO, Marisa Ferreira; ARAÙJO, Vera Lúcia Santiago; DANTAS, João Francisco de Lima; MASCARENHAS, Renata de Oliveira; ALVES, 
Jefferson Fernandes (Org.). Pesquisas teóricas e práticas em audiodescrição. 1. ed. Natal: EDUFRN, 2016, v. 1. p. 00-00.

TOURY, Gideon. Descriptive Translation Studies and Beyond. Amsterdam/Philadelphia: John Benjamins, 1995/2012. doi: $\underline{10.1075 / \mathrm{btl} .4}$

VAN DOORSLAER, Luc; FLYNN, Peter; LEERSSEN, Joep (Org.). Interconnecting Translation Studies and Imagology. Amsterdam/Philadelphia: John Benjamins, 2016.

Recebido: 25/06/2019

Aceito: 28/02/2019

Publicado: 01/07/2019 